\title{
Prediction schemes to enhance the routing process in geographical GPSR ad hoc protocol
}

\author{
Raed Saqour ${ }^{\mathrm{a}}$, Mohamad Shanuldin ${ }^{\mathrm{a}}$ and Mahamod Ismail ${ }^{\mathrm{b}}$ \\ ${ }^{a}$ Faculty of Science and Information Technology, Universiti Kebangsaan Malaysia Bangi, Selangor, \\ 43600, Malaysia \\ E-mail: r_saqour2002@yahoo.com;msz@ftsm.ukm.my \\ ${ }^{\mathrm{b}}$ Faculty of Engineering, Universiti Kebangsaan Malaysia Bangi, Selangor, 43600, Malaysia \\ E-mail: mahamod@eng.ukm.my
}

\begin{abstract}
Geographical routing protocols have received a serious attention due to more advantages they have in comparison to the conventional routing protocols. They require information about the physical position of nodes needed to be available. Commonly, each node determines its own position through the use of Global Positioning System (GPS) or some other type of positioning service. Greedy Perimeter Stateless Routing (GPSR) protocol, which is one of geographical routing protocols, limits the forwarding decision of the packet based on the node's own position, the destination's position and the position of the forwarding node's neighbors. Location information has some inaccuracy depending on the localization system and the environment exists in. This paper aims to study the impact of mobility metrics (beacon interval, and node speed) on introducing location information error in GPSR protocol using different mobility models. The effect of these metrics is identified in GPSR as Neighbor Break Link (NBL) problem. Based on simulation analysis, mobility prediction schemes are proposed to migrate the observed problem.
\end{abstract}

\section{Introduction}

Geographical routing protocols $[3,16]$ in ad hoc networks have received serious attention due to their substantial advantages as compared to topology-based routing protocols [5], being either proactive or reactive protocols. Geographical routing protocols eliminate some of the limitations of topology-based routing by using location information.

In geographical routing protocols, the packet forwarding decision by a node is primarily based on the position of a packet's destination and the position of the node's immediate one-hop neighbors. Typically, the packet is forwarded to the neighbor which is closer to the destination than the forwarding node itself. Thus, that makes more progress toward the destination.

Although the position-based routing is a key element in providing a scalable solution for routing in ad hoc network [17], an important requirement for any position-based routing protocol is the needed for accurate position information of the nodes. Thus, the nodes in position-based routing protocols need to maintain up-to-date positions of their immediate neighbors in their neighbors list for making effective routing decisions. Due to that, each node periodically within a time interval broadcasts a short beacon packet to announce its presence and position to its neighbors with its transmission range. Receiving 
nodes list all known neighbor nodes along with their position in the neighbors list and remove entries after they have failed to receive a beacon for a certain time from the corresponding node.

Due to the node mobility, the network topology changes frequently especially in high dynamic network like ad hoc, the neighbors' relation changes frequently and local topology rarely remains static. Using periodic beaconing has several drawbacks such as unnecessary utilization of network resources, consumption of scares battery power in addition to inaccuracies in the node position.

In this paper, we analyze the effect of mobility metrics; beacon interval and node speed, in introducing a location error in GPSR geographical routing protocol. Based on the simulation results, Neighbor Break Link (NBL) problem which is due to link break connection with neighboring nodes is identified and discussed. In addition, we provide remedies for the identified NBL problem with a suggested mobility prediction schemes.

We examine the following two main mobility metrics that cause the NBL problem and affect the performance of GPSR routing protocol:

- Beacon Interval: it is not possible to avoid the time gap between the measurement of a location and the time when the information is actually looked up for routing decision. This is because the time interval between location updates is generally longer than the inter-packet arrival times.

- Node speed: mobile node can move in different speed and the maximum node speed is a critical cause for the NBL problem.

The rest of the paper is organized as follows. In Section 2, we provide background and the related works. In Section 3, neighbor break link problem is identified. In Section 4, the effect of mobility metrics; beacon interval and node speed, as a cause to NBL problem in GPSR routing protocol is analyzed and discussed. In Section 5, mobility prediction schemes and simulation result are introduced, and Section 6 introduces conclusion and a future work for the paper.

\section{Background and related works}

\subsection{Greedy Perimeter Stateless Routing (GPSR) protocol}

GPSR protocol $[1,16,10]$ is an efficient, localized routing algorithm in large-scale ad hoc wireless networks. Under GPSR protocol, a node makes routing decisions only based on the locations of its (one-hop) neighbors and the location of the destination node available by using location service protocol like Grid Location Service (GLS), GPSR thereby avoids the overhead of maintaining global topology information.

GPSR makes greedy forwarding decisions using only information about the position of immediate neighbors in the network topology as it appears in Fig. 1. In Fig. 1, $x$ receives a packet destined to $D$, $x$ 's radio range is denoted by the dotted circle about $x$, and the arc with radius which is equal to the distance between $y$ and $D$ is shown as the dashed arc about $D$. $x$ forwards the packet to $y$ as $y$ listed in $x$ neighbors list as shown in Table 1 and as the distance between $y$ and $D$ is less than that between $D$ and any off $x$ 's other neighbors. This greedy forwarding process is repeated by nodes $y, k, z$, and $w$ until the packet reaches the destination node $D$.

The power of greedy forwarding to route using only neighbor nodes' positions comes with one attendant drawback. There are topologies in which the only route to a destination requires a packet move temporarily farther in geometric distance from the destination. A simple example of such a topology is shown in Fig. 2. Here, $x$ is closer to $D$ than its neighbors $w$ and $y$. Although two paths; $(x-y-z-D)$ 
Table 1

Node $x$ neighbors list

\begin{tabular}{cc}
\hline Node-id & Neighbor $(\mathrm{x}, \mathrm{y}$ coordinates $)$ \\
\hline $\mathrm{A}$ & $\mathrm{A}(\mathrm{x}, \mathrm{y})$ \\
$\mathrm{B}$ & $\mathrm{B}(\mathrm{x}, \mathrm{y})$ \\
$\mathrm{C}$ & $\mathrm{C}(\mathrm{x}, \mathrm{y})$ \\
$\mathrm{F}$ & $\mathrm{F}(\mathrm{x}, \mathrm{y})$ \\
$\mathrm{y}$ & $\mathrm{y}(\mathrm{x}, \mathrm{y})$ \\
\hline
\end{tabular}

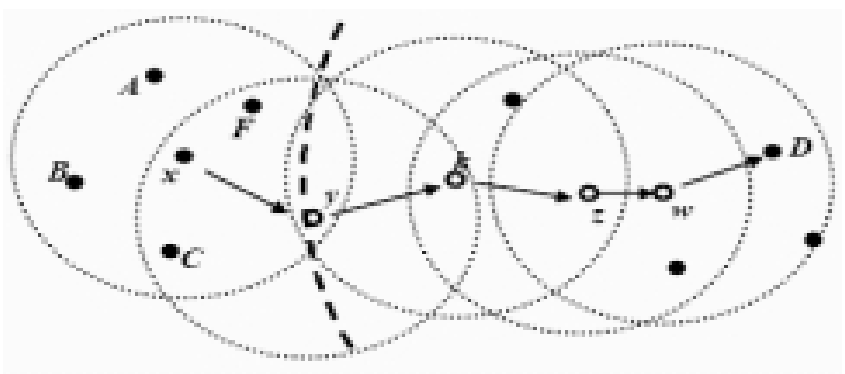

Fig. 1. Greedy forwarding.

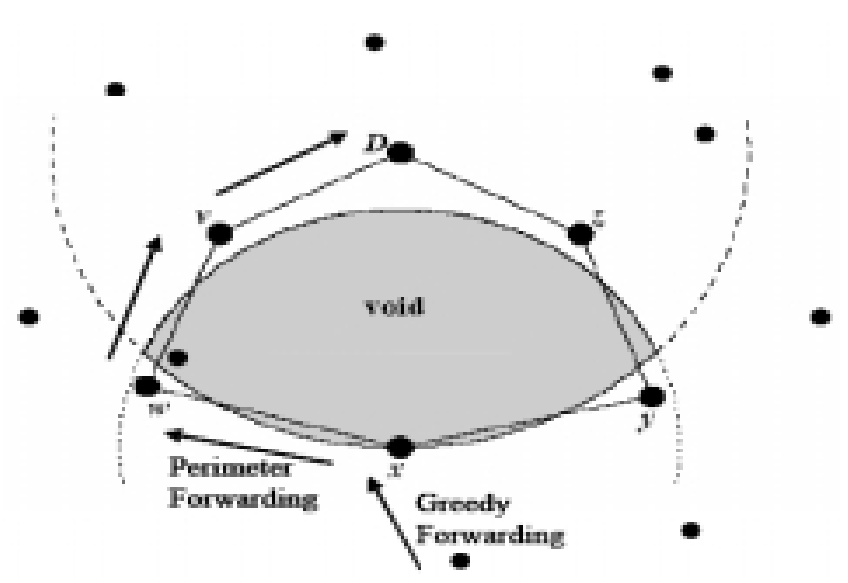

Fig. 2. Perimeter forwarding.

and ( $x-w-v-D$ ) exist to $D, x$ will not choose to forward to $w$ or $y$ using greedy forwarding. The protocol terms $x$ as local maximum in its proximity to $D$ and terms shaded region without nodes as void region, then it uses perimeter forwarding strategy to forward the packet around void region.

In perimeter forwarding, the protocol constructed planarized graph ${ }^{1}$ for the nodes' neighbors and applied the right-hand rule to route the packet around void region. The right-hand rule states that when arriving at node $x$ from $y$, the next traversed edge is the next one sequentially counterclockwise about $x$ from edge $(x, y)$.

Network connectivity in GPSR protocol is determined by simple beacon packet protocol which provides all nodes with their neighboring positions. Periodically, each node transmits a beacon packet containing

\footnotetext{
${ }^{1}$ Planar graph is a graph that can be drawn in the plane with no crossing edges.
} 


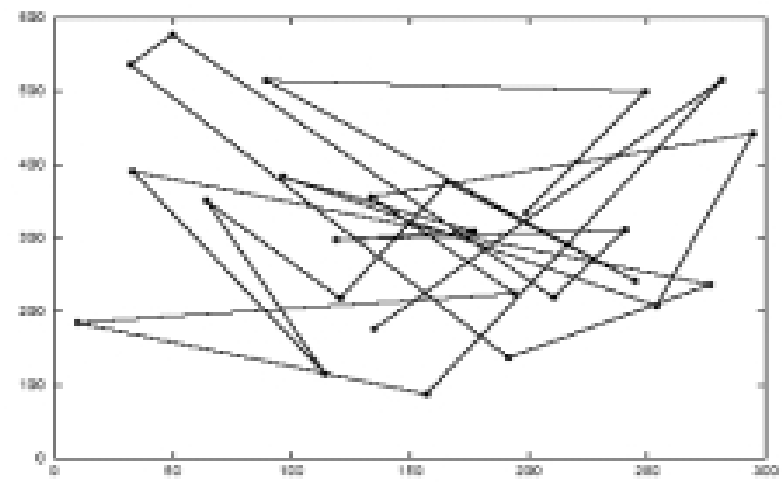

(a)

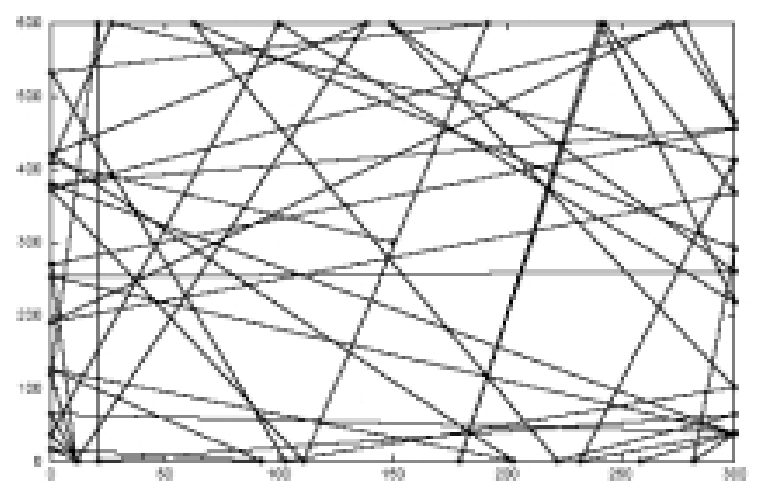

(b)

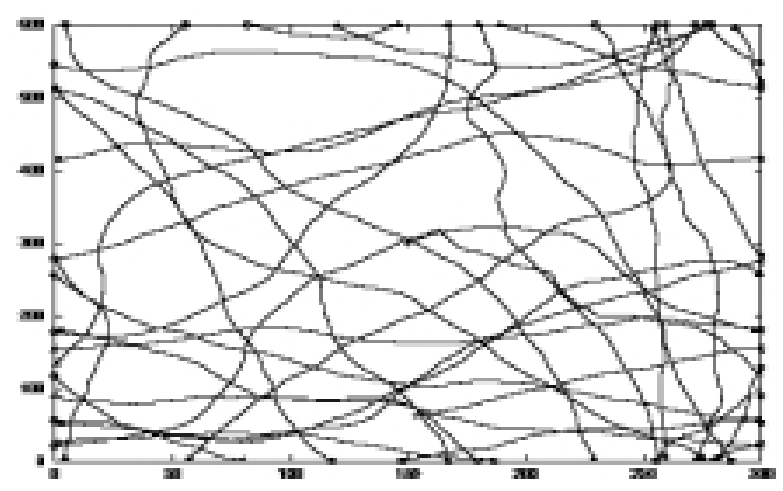

(c)

Fig. 3. Mobility models: (a) Random Waypoint. (b) Random Direction. (c) Bound Simulation Area.

only its own identifier (e.g., IP address) and its position, when any node receives a beacon packet from its neighbors; it creates or refreshes its neighbors list and uses this beacon packet information for future routing process.

\subsection{Mobility models}

We adopt a rich set of mobility models for our study: RW, RD, and BSA mobility model [19]. Each one was chosen to create certain Mobile Nodes (MNs) movement pattern that is not captured by others.

1. Random Waypoint $(R W)$. RW mobility model as shown in Fig. 3(a) includes pause times between changes in direction and/or speed. A MN begins by staying in one location for a certain period of time (i.e., a pause time). Once this time expires, the MN chooses a random destination in the simulation area and a speed that is uniformly distributed between 0 and some maximum speed. The $\mathrm{MN}$ then travels toward the newly chosen destination at the selected speed. Upon arrival, the MN pauses for a specified time period before starting the process again.

2. Random Direction (RD). In RD as shows in Fig. 3(b), MN chooses a random direction in which to travel similar to the Random Waypoint mobility model. A MN then travels to the boarder of the simulation area in that direction. Once the simulation boundary is reached, the MN pauses for a 
specified time, chooses another angular direction (between 0 and 360 degrees) and continues the process.

3. Bound Simulation Area (BSA). In BSA mobility model as shows in Fig. 3(c), a relationship between the previous direction of travel and velocity of a MN with its current direction of travel and velocity exists. A velocity vector $v=(v, \theta)$ is used to describe MN's velocity $v$ as well as its direction $\theta$; the MN's position is represented as $(x, y)$. Both the velocity vector and the position are updated at every $\Delta t$ time steps according to the following formulas:

$$
\begin{aligned}
& v(t+\Delta t)=\min [\max (v(t)+\Delta v, 0), \text { Vmax }] \\
& \theta(t+\Delta t)=\theta(t)+\Delta \theta \\
& x(t+\Delta t)=x(t)+v(t) * \cos \theta(t) \\
& y(t+\Delta t)=y(t)+v(t) * \sin \theta(t)
\end{aligned}
$$

where Vmax is the maximum velocity defined in the simulation, $\Delta v$ is the change in velocity which is uniformly distributed between [-Amax* $\Delta t, A \max * \Delta t]$, Amax is the maximum acceleration of a given $\mathrm{MN}, \Delta \theta$ is the change in direction which is uniformly distributed between $\left[-\alpha^{*} \Delta t, \alpha^{*} \Delta t\right]$, and $\alpha$ is the maximum angular change in the direction of MN traveling.

\subsection{Related works}

The first and most significant contribution to prediction in ad hoc routing to date has been the work by Su [18]. In Su's approach, a Route Expiration Time (RET), which is the estimated time when the route between two nodes is predicted to terminate, is calculated for each source-destination pair. The advantages of this approach are primarily that there is only a small computational and bandwidth overhead required for such approach while the disadvantage is the signal propagation environment are not discussed and the lack of use of future node movement information.

In [4], the authors proposed analytical solution model similar to Su's approach, by exploring the expected lifetime of a route. In this approach, the prediction of the lifetime of a route is possible when the destination node knows the location and velocity of all nodes along the route. As with Su's approach, the effects of the signal propagation environment are not discussed, nor is any signal loss map proposed. The authors note that a completely deterministic mobility pattern is highly improbable, and do not consider autonomous nodes. This work needs also to be investigated by simulation and within real ad hoc routing protocols to prove its effectiveness.

The authors in [6] proposed different approach by using signal loss map to generate a prediction. This signal loss map is generated in real time with the ability to continually update and propagate the map, as well as vary its level of detail depending on the environment. This map presents a logical view of signal propagation over a physical coordinate system, mapping areas of varying signal loss as they are discovered by nodes. The advantage of this approach is that a more accurate view of the signal propagation environment is available, and consequentially the potential for better predictions to be made. The disadvantages are that map sharing adds bandwidth overhead and map development adds a small amount of computational overhead.

Different approach attempted in [7] by taking into consideration that each node's predicted future mobility information. The solution is known as the Future Neighbors Table (FNT). The FNT provides routing algorithms with a list of times when pairs of nodes will gain and lose the quality of being communication neighbors. Routing algorithms thus have an extended prediction period into the future. 
Routing algorithms also have access to the possibility of when routes will come into existence in the future, though this aspect has not been applied to date. Although future node mobility sharing in autonomous networks may likely be required anyway in such networks, it is a bandwidth overhead over previous methods. There is also a significant computational overhead for calculating the FNT.

In [13], the authors formulate and study the reliability routing problem in ad hoc network. They use waypoint graph to model the working area of ad hoc network and present an offline algorithm to compute a duration prediction table for the given waypoint graph. In order for their prediction algorithm to work, the authors made many assumptions in working area and the presence of waypoint graph to model the ad hoc area increases the complexity of the system.

In previous studies, the authors are also concerned about link break that occurs between the source and destination. In addition to, their prediction schemes/algorithms suffer from bandwidth, communication and computational overhead. In this paper, the distinction from previous studies is the isolation of the neighbor break link effects of neighboring nodes. However, we are not concern with the route breaks link effects between the source and the distention node caused by intermediate nodes that other studies concern about. We also concentrate in our study on geographical routing protocols presented by GPSR protocol which has not been investigated like other proactive or reactive routing protocols. With our mobility prediction schemes, no overhead communication added to the network

In [14], the authors summarized the mobility metrics that impact the routing protocols in mobile ad hoc network. These mobility metrics are the concern of our study. An analysis on the effect of mobility metrics on NBL problem is conducted. Only the considered neighboring nodes are those within the node transmission range. Derived NBLP scheme is proposed according to our observations.

\section{Neighbor break link problem}

In GPSR protocol, every node periodically within a time interval, broadcasts a beacon packet within its own radio range. The beacon packet carries the node-id and the current location information $(x, y)$ coordinates of the node. Every node, which receives a beacon packet within its transmission range creates a new entry into its neighbors list for the incoming node beacon packet and hence keeps information carried within incoming beacon packet on its neighbors list for later use in a route process. In this way, all the nodes in the network will have the geographical location information about their neighbor nodes within its transmission range.

When the sender node wants to send a packet to a destination, it searches it's neighbors list looks for the node that is closer to destination node. However, the selected next hop node may not exist within the radio range of the sender while it is listed as a neighbor in the neighbors list. This situation is defined as Neighbor Break Link (NBL) problem and can be affected by high node speed, and long beacon interval. When a node moves outward of the intended receiver, connection between the sender and the receiver can be broken.

Figure 4 depicts the NBL problem; GPSR protocol defined the node $y$ as a forwarding node since it is listed in neighbors list of node $x$. that is because of the beacon packet heard from node $y$ by node $x$ at time $t_{1}$ and as it closer to destination node $D$. However, because of the node speed and beacon packet interval of node $y$, when node $x$ decides to forward the packet to $y$ at time $t_{2}$, node $y$ may will be in location $L_{y 2}$ which is not recognized by node $x$ at the time of forwarding decision of the packet decided by node $x$, this that would lead to the dropping of a packet and effect the packets delivery and system throughputs performance. 


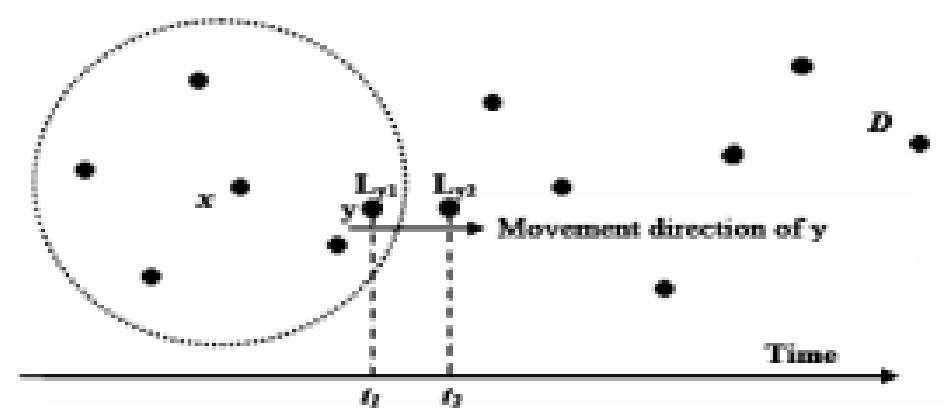

Fig. 4. Neighbor Break Link (NBL) problem. $t_{1}$ : time instance of $y$ last location heard by node $x, t_{2}$ : time of packet forward decision made by node $x$.

\section{Mobility metrics effect in GPSR protocol}

In [14], the authors classified various mobility metrics that can affect routing protocols on mobile ad hoc network. In this paper, we investigate the influence of some of these mobility metrics on NBL problem in GPSR protocol.

After a careful consideration of simulation environments like NS-2 and GloMoSim, it is decided to build a discrete event simulator from the scratch using Borland $\mathrm{C}++$. That is since none of the above mentioned simulators could provide with BSA mobility model needed on this study. In addition, none of them give us the facilities to trace in details all the simulator behaviors.

To estimate the effect of inaccurate location information caused by node mobility metrics on the geographic routing protocol, we conducted simulation study varying the beacon interval and the maximum node speed. GPSR protocol is selected for our simulation because it is shown to perform correctly and efficiently with the exact location information compared with other routing protocols. We use selected simulation parameters similar to those used in [1], 100 nodes are placed randomly in a $1500 \mathrm{~m} \times 300 \mathrm{~m}$ field and the combination of beacon intervals of $0.1,0.25,0.5,1.0,3.0,4.0,5.0 \mathrm{sec}$ and maximum node speed of $10,20,30,40,50 \mathrm{~m} / \mathrm{sec}$ are simulated. To filter out the noise in simulation results, ten different scenarios are generated for each distinct parameter setting and the results represent the average value.

We evaluate the performance of GPSR protocol based on the following metrics:

- Packet Drop-Neighbor Break: the number of data packets drop due to the link breaks between the nodes and there neighbors during the packet forwarding process.

- Delivery Ratio: The number of successfully data packets delivered to destination over the total number of packets transmitted.

- End-to-End Delay: the average time delay for data packets from the source node to destination node.

\subsection{Effect of beacon interval}

To maintain connectivity in GPSR protocol, a node upon not receiving a beacon packet from a neighbor for longer than specific time interval assumes that the neighbor is no longer within transmission range and connectivity has been lost. One of the variables that control the determination of network connectivity is a beacon packet interval [11]. Beacon packet interval specifies the maximum time interval between the transmissions of beacon packets among the nodes. The position that a node associates with a neighbor becomes less current between beacon packets as that neighbor moves. The accuracy of the neighbors' 


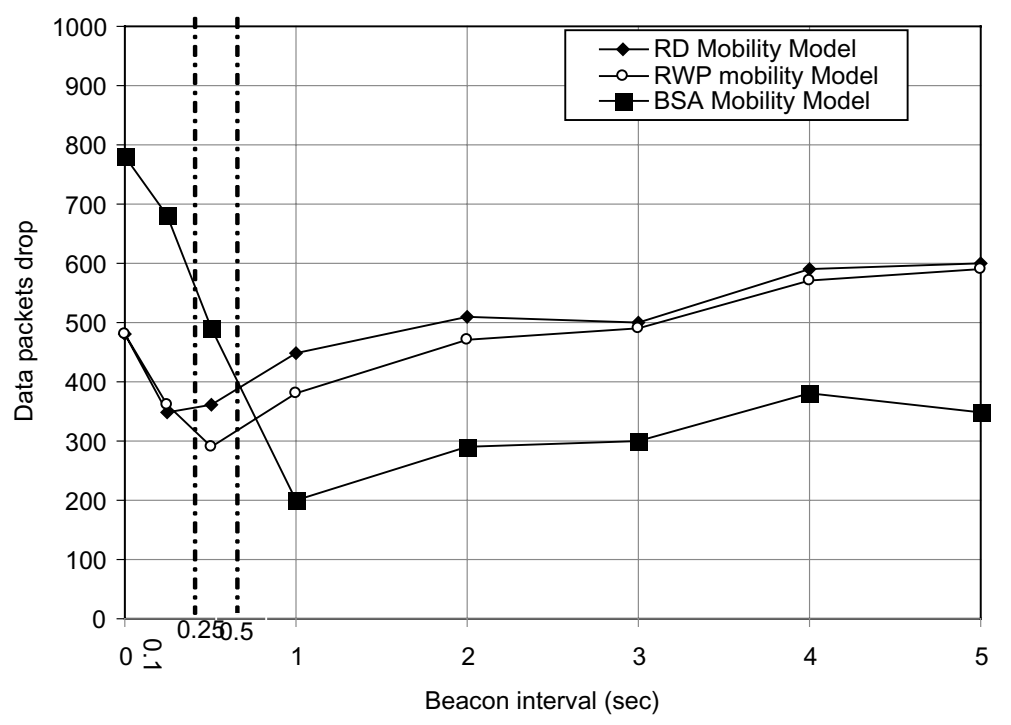

Fig. 5. Beacon interval effect.

Locations also decreases; old neighbors may leave and new neighbors may enter radio transmission range without recognizing that from nodes.

Figure 5 that shows the number of packet drops increases as the beacon intervals increase for all mobility models examined, RD and RW mobility models performs best (minimum packets drop) during the beacon interval $0.5 \mathrm{~s}$ while BSA performs best during the beacon interval $1.0 \mathrm{~s}$. When we compare the reason for packet drops before these beacon intervals $(0.5 \mathrm{~s}, 1.0 \mathrm{~s})$, we found that much more packets drop are caused by the node buffer overflow rather than by the beacon intervals. While the packets dropping in beacon intervals $0.5 \mathrm{~s}, 1.0 \mathrm{~s}$ and greater caused by NBL problem and its increases with increasing in beacon interval time.

Figure 6 shows that the number of beacon packets drop is caused by node buffer overflow for all examined mobility models. In RW and RD mobility models, congestion caused by more beacon packets deceases until it reaches its minimum value in beacon interval $0.5 \mathrm{~s}$ while in BSA in value $1.0 \mathrm{~s}$. The result shows that frequent beacon packets will not improve the performance of the protocol but it will make congestion in the network and waste the network resources. This is contrast to what is suggested in [15] for beacon interval to be very short.

During the simulation, some interesting results are observed. BSA mobility model can not be judged to have the best performance related to NBL problem in beacon interval $1.0 \mathrm{~s}$, nodes mobility pattern using BSA have another effect in NBL occurrence.

Figure 7 shows BSA mobility model with different nodes movement patterns caused by changing the direction traveling of the MNs. When we increase the direction $\theta$ of MNs movement, the nodes start to move closer to each other. That will cause the neighbor nodes to receive many beacon packets from each other and will cause the node buffers to overflow quickly in small beacon interval. In addition to, increasing the direction $\theta$ of MNs movement will cause the NBL to occur less since the nodes will not intend to break the link with their neighbors frequently.

Figure 8 shows the effect of changing the direction degree for different $\theta(30,90,180$, and 270). The best performance of BSA mobility model in direction 30 is in beacon interval $1.0 \mathrm{~s}$, while in the directions $90,180,270$ is in beacon intervals $1.4 \mathrm{~s}, 2.0 \mathrm{~s}$ and $2.8 \mathrm{~s}$ respectively. This is due to the nodes movement pattern taken in different direction. 


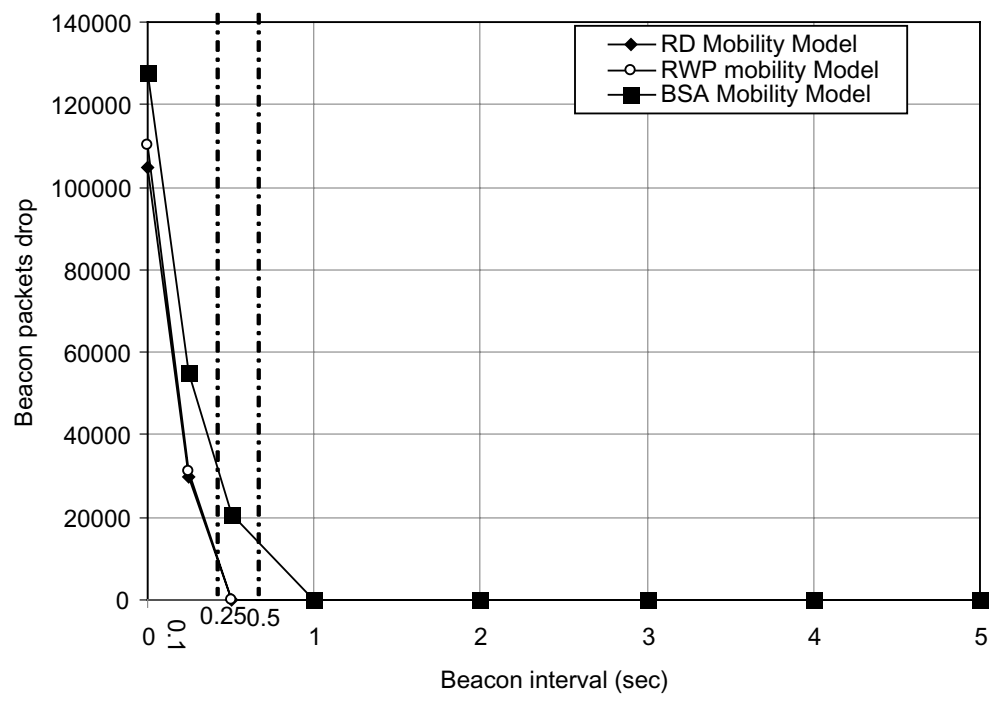

Fig. 6. Beacon packet drops varying beacon interval.

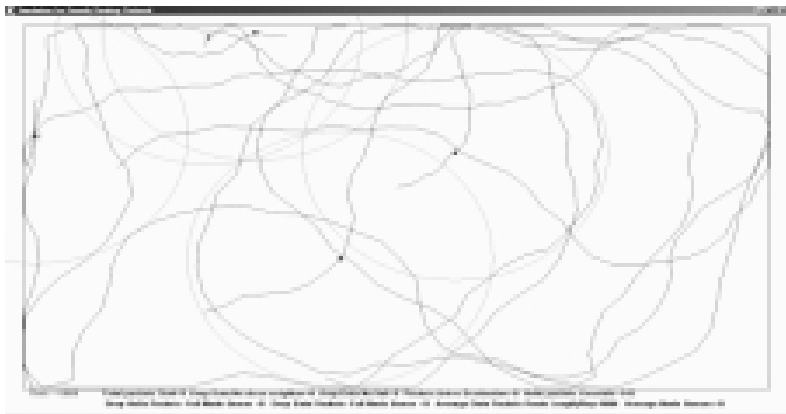

(a). 30 degree

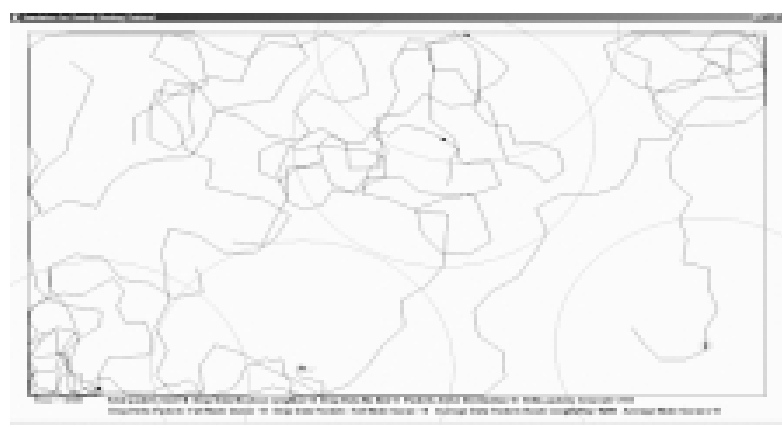

(c). 180 degree

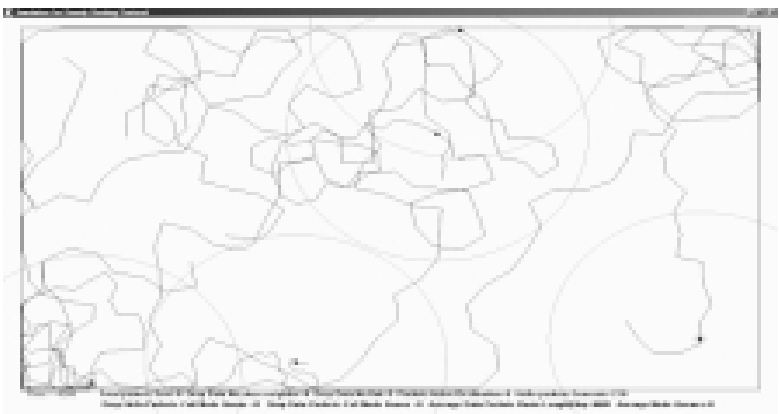

(b). 90 degree

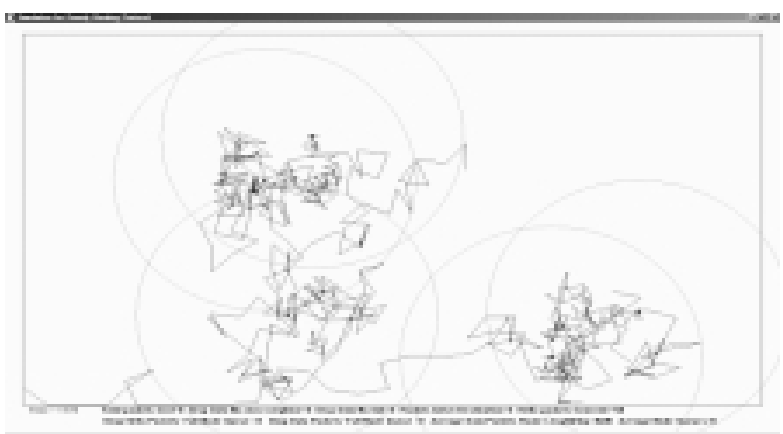

(d). 270 degree

Fig. 7. BSA nodes movement with different degree.

\subsection{Effect of node speed}

Variation of the node speed means a change in the degree of node mobility which in turn affects the error in node location information error and increase the NBL problem occurrence. The performance of 


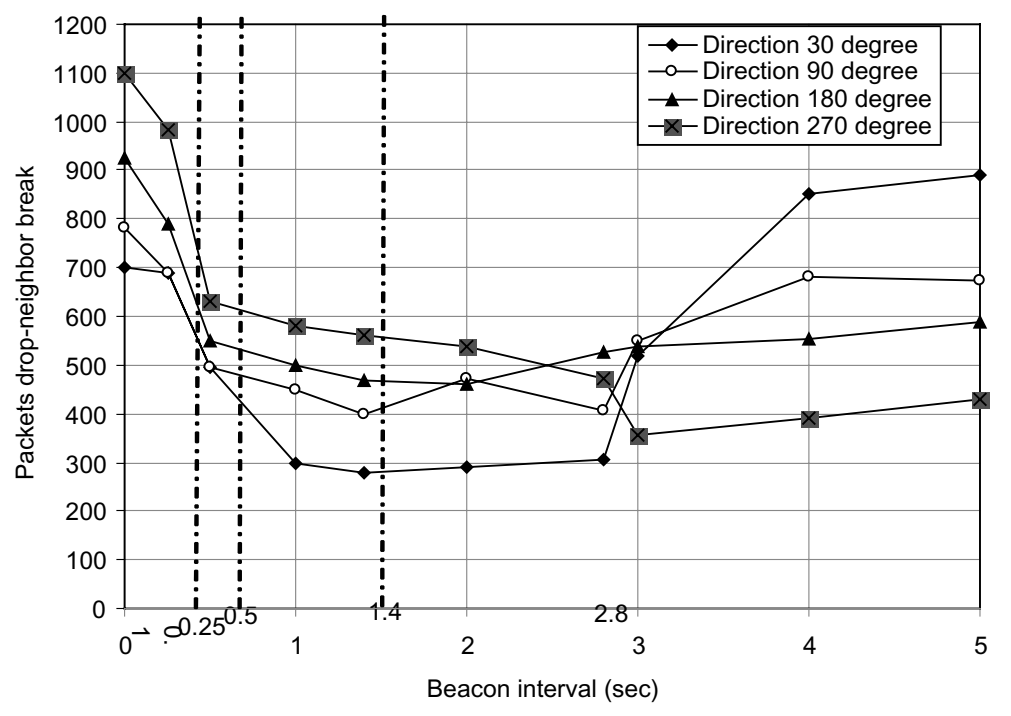

Fig. 8. Data packets drop varying beacon interval (BSA mobility model).

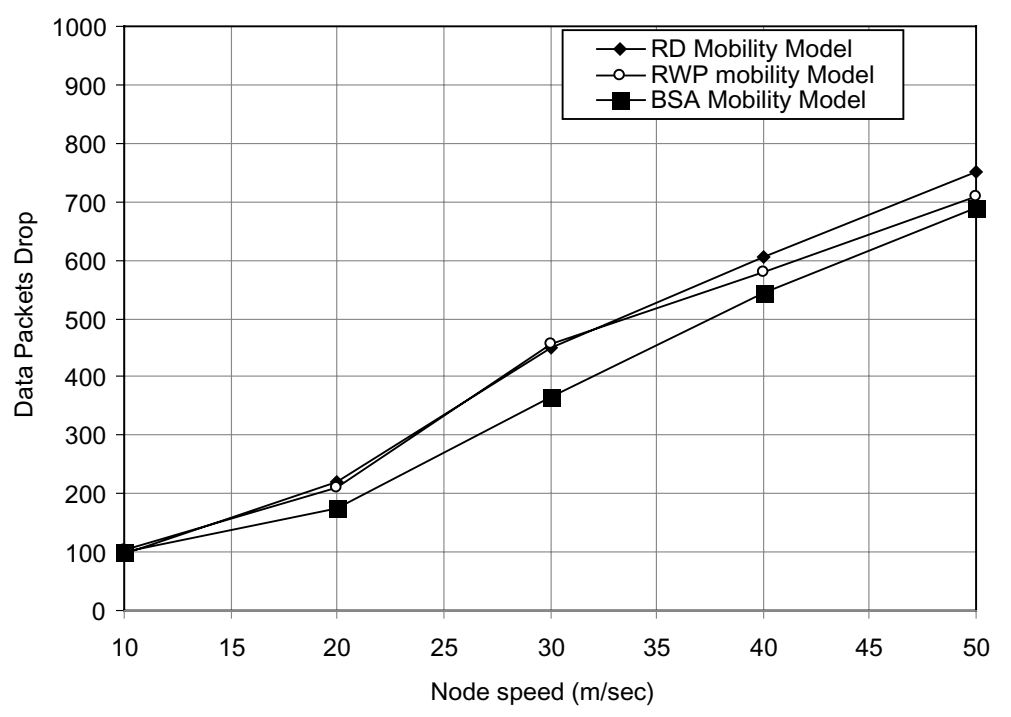

Fig. 9. Node speed effect.

GPSR protocol is fully related to the accuracy of node location which is effected by node speed.

Figure 9 shows the effect of node speed on the performance of GPSR protocol. The general effect of node speed on the performance of GPSR protocol is closely similar to all tested mobility models. The number of packet drops caused by NBL increases as the maximum node's speeds increases. BSA mobility model performs better than other mobility models with less packets drop, as we can intuitively expect from the neighbors movement pattern of the nodes in BSA. 
Table 2

The notations for mobility prediction scheme 1

\begin{tabular}{cl}
\hline Variables & Definition \\
\hline$t_{2}, t_{1}$ & Time instance of node position where $t_{2}>t_{1}$ \\
$\left(x_{t_{1}}^{i}, y_{t_{1}}^{i}\right)$ & The coordinate of node $i$ along the direction of the $x$ and $y$ axes at time $t_{1}$ \\
$\left(x_{t_{2}}^{i}, y_{t_{2}}^{i}\right)$ & The coordinate of node $i$ along the direction of the $x$ and $y$ axes at time $t_{2}$ \\
$v_{t_{2}}^{i}$ & The velocity of node $i$ at time $t_{2}$ \\
$T_{c}$ & The current time \\
$\left(x_{p}^{i}, y_{p}^{i}\right)$ & The predicted position of node $i$ at time $T_{c}$ \\
\hline
\end{tabular}

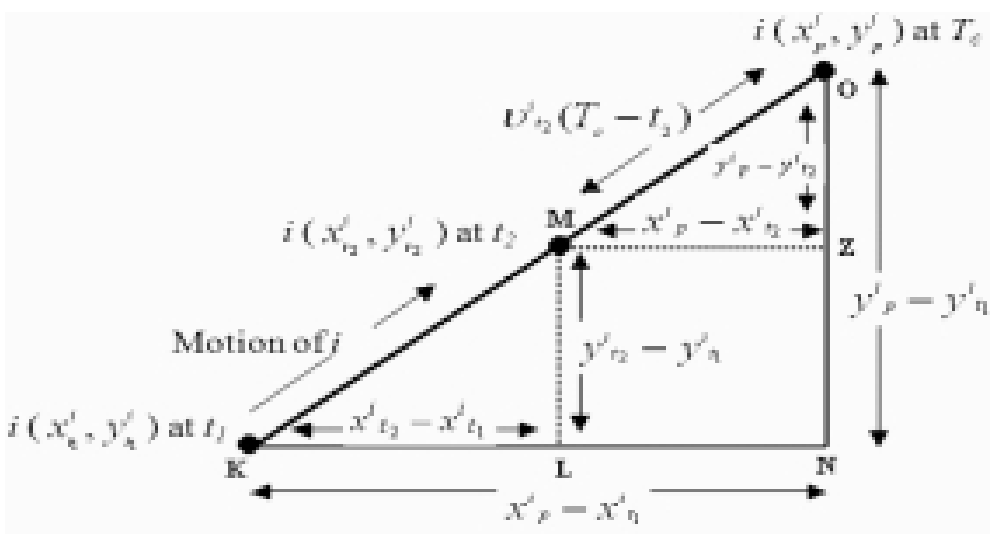

Fig. 10. Prediction of node B location at a given time in the future using mobility prediction scheme 1.

\section{Mobility prediction schemes}

In this section, the suggested mobility prediction schemes along with the simulation results for GPSR protocol to overcome NBL problem are presented and discussed. The proposed schemes are based on the physical concept of point's movements observed in [9]. The proposed prediction schemes do not require any additional communication between the nodes at the network.

\subsection{Mobility prediction scheme 1}

In order to enhance the proposed prediction scheme 1 to work, we need additional fields on the node neighbors list: we need two $x, y$ co-ordinates for the neighbor nodes and beacon packet time when these $x, y$ co-ordinates reach. In addition, the second $x, y$ co-ordinate also indicates $v$ to be the velocity of neighbor nodes at the second neighbor nodes location. Table 2 illustrates the notations used for mobility prediction scheme 1 .

For now, we assume that the node move in a piecewise linear pattern. In other words, it is assumed that between successive update beacon packets, the nodes move in a straight line. In a piecewise linear motion pattern, two pervious updates are sufficient to predict a future location of the mobile nodes-in-the-plane.

Let $x_{t_{1}}^{i}, y_{t_{1}}^{i}$ and $x_{t_{2}}^{i}, y_{t_{2}}^{i}$ where $t_{2}>t_{1}$ be the latest two updates (beacon packet information) respectively from a neighbor node $i$ to a particular correspondent node $A$, and let $v_{t_{2}}^{i}$ be the velocity of node $i$ at location $x_{t_{2}}^{i}, y_{t_{2}}^{i}$. Assume also node $A$ wishes to predict the location $x_{p}^{i}, y_{p}^{i}$ of node $i$ at some instant time $T_{c}$ (at time node $A$ decides to forward a packet to $i$ ). This situation is depicted in Fig. 10. 
From Fig. 10, by using the similarity of triangles $K L M$-and-KNO, we-get:

$$
\frac{y_{t_{2}}^{i}-y_{t_{1}}^{i}}{y_{p}^{i}-y_{t_{1}}^{i}}=\frac{x_{t_{2}}^{i}-x_{t_{1}}^{i}}{x_{p}^{i}-x_{t_{1}}^{i}}
$$

If we want to predict the location of node $i$ at y-axis, we can solve for $y_{p}^{i}$ from Eq. (5),

$$
y_{p}^{i}=y_{t_{1}}^{i}+\frac{\left(x_{p}^{i}-x_{t_{1}}^{i}\right)-\left(y_{t_{2}}^{i}-y_{t_{1}}^{i}\right)}{\left(x_{t_{2}}^{i}-x_{t_{1}}^{i}\right)}
$$

Using Eq. (6), node $A$ can predict the location of node $i$ at y-axis $y_{p}^{i}$ if it knows $x_{p}^{i}$, which in turn can be calculated as follows, using similarity of triangles $K L M$ and $M Z O$ from Fig. 10, we get:

$$
\frac{y_{t_{2}}^{i}-y_{t_{1}}^{i}}{y_{p}^{i}-y_{t_{2}}^{i}}=\frac{x_{t_{2}}^{i}-x_{t_{1}}^{i}}{x_{p}^{i}-x_{t_{2}}^{i}}
$$

If we use Pythagors' theorem at the triangle $M Z O$, we get:

$$
v_{t_{2}}^{i}{ }^{2}\left(T_{c}-t_{2}\right)^{2}=\left(x_{p}^{i}-x_{t_{2}}^{i}\right)^{2}+\left(y_{p}^{i}-y_{t_{2}}^{i}\right)^{2}
$$

Substituting for $y_{p}^{i}-y_{t_{2}}^{i}$ from Eq. (7) into Eq. (8), we get:

$$
v_{t_{2}}^{i}{ }^{2}\left(T_{c}-t_{2}\right)^{2}=\left(x_{p}^{i}-x_{t_{2}}^{i}\right)^{2}+\left[\frac{\left(y_{t_{2}}^{i}-y_{t_{1}}^{i}\right)\left(x_{p}^{i}-x_{t_{2}}^{i}\right)}{\left(x_{t_{2}}^{i}-x_{t_{1}}^{i}\right)}\right]^{2}
$$

by Solving $x_{p}^{i}$ in Eq. (9), we get:

$$
x_{p}^{i}=x_{t_{2}}^{i}+\left[\frac{v_{t_{2}}^{i}\left(T_{c}-t_{2}\right)\left(x_{t_{2}}^{i}-x_{t_{1}}^{i}\right)}{\left[\left(x_{t_{2}}^{i}-x_{t_{1}}^{i}\right)^{2}+\left(y_{t_{2}}^{i}-y_{t_{1}}^{i}\right)^{2}\right]^{1 / 2}}\right]
$$

\subsection{Mobility prediction scheme 2}

The previous prediction scheme works well if we have two $x, y$ coordinates position based information for the neighbor nodes. In some scenario, these two points may not be available for the nodes to predicate their neighbor's future position. Consider the example illustrated in Fig. 11, where node $i$ moves from position $P_{1}$ to $P_{2}$. Now, assume that according to node $i$ beacon interval, it will send its beacon packet at position $P_{x}$. In this scenario, node $A$ will receive only one $\mathrm{x}, \mathrm{y}$ coordinate position information from node $i$ and node $A$ will be miss leading if it needs to use mobility prediction scheme 1 to predicate the future position of node $i$ while its forwarding the data to destination $D$. For that, we need another scheme to overcome the drawback of mobility prediction scheme 1 as it is illustrated on Fig. 11.

Let us assume that the latest update (beacon packet information) was generated at time $t_{1}$ from node $i$ at location $x_{t_{1}}^{i}, y_{t_{1}}^{i}$, reporting a velocity $v_{t_{1}}^{i}$ and direction $\theta_{t_{1}}^{i}$. By that, the node moves at an anti-clockwise angle $\theta_{t_{1}}^{i}$ to horizontal. Let the velocity $v_{t_{1}}^{i}$ and direction $\theta_{t_{1}}^{i}$ at the time $T_{c}$ remain unchanged since the last update. Assume that a node $A$ wishes to predict the location $x_{t_{1}}^{i}, y_{t_{1}}^{i}$ of node $i$ at some instant time $T_{c}$ (at time node $A$ decides to forward a packet to $i$ ). This situation is depicted in Fig. 12 and Table 3 in which the notations used for mobility prediction scheme 2 are illustrated. 
Table 3

The notations for mobility prediction scheme 2

\begin{tabular}{cl}
\hline Variables & Definition \\
\hline$t_{1}$ & Time instance of node position \\
$\left(x_{t_{1}}^{i}, y_{t_{1}}^{i}\right)$ & The coordinate of node $i$ along the direction of the $x$ and $y$ axes at time $t_{1}$ \\
$v_{t_{2}}^{i}$ & The velocity of node $i$ at time $t_{1}$ \\
$\theta_{t_{1}}^{i}$ & The node direction with respect to x-axis \\
$T_{c}$ & The current time \\
$\left(x_{p}^{i}, y_{p}^{i}\right)$ & The predicted position of node $i$ at time $T_{c}$ \\
\hline
\end{tabular}

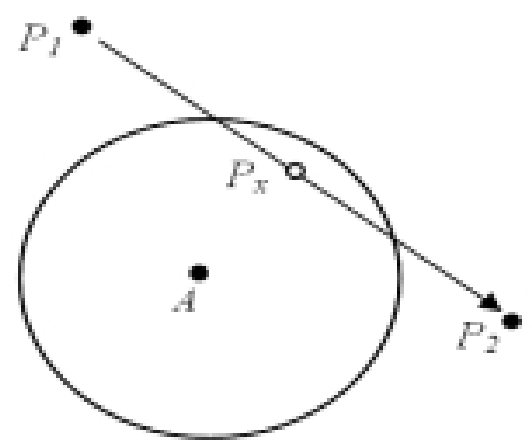

Fig. 11. An example illustrating the drawback of mobility prediction scheme 1 .

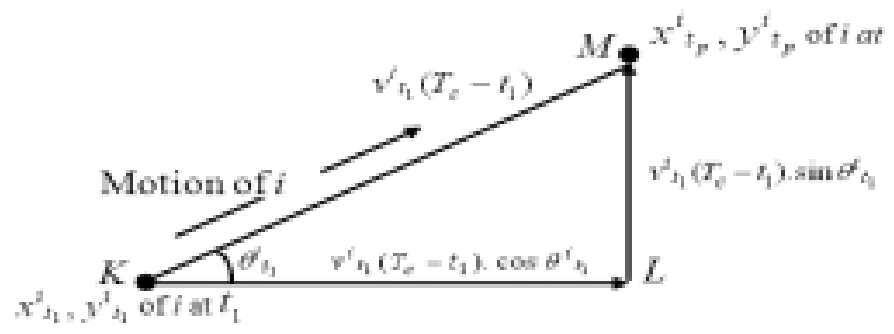

Fig. 12. Prediction of node i location at a given time in the future using mobility prediction scheme 2 .

From Fig. 12, applying laws of sins and cosines on triangle $K L M$, we-get:

$$
\begin{aligned}
& x_{p}^{i}=x_{t_{1}}^{i}+v_{t_{1}}^{i}\left(T_{c}-t_{1}\right) \cdot \cos \theta_{t_{1}}^{i} \\
& y_{p}^{i}=y_{t_{1}}^{i}+v_{t_{1}}^{i}\left(T_{c}-t_{1}\right) \cdot \sin \theta_{t_{1}}^{i}
\end{aligned}
$$

By using Eqs (11) and (12), any node can predict the location of node $i$ within its transmission range at $\mathrm{x}$-axis $x_{p}^{i}$ and y-axis $y_{p}^{i}$ once it knows the latest $x_{t_{1}}^{i}, y_{t_{1}}^{i}$ location, the velocity $v_{t_{1}}^{i}$, direction of motion $\theta_{t_{1}}^{i}$, and the time $t_{1}$ for these values arriving time are known.

In summary, When the source node has a packet to be forwarded to a destination, it first uses the information available in it's neighbors list to predict the location of it's neighbor nodes by using Eqs (6) and (10) if it has two $x, y$ coordinates position based information for its neighbors or by using Eqs (11) and (12) if the node has only one $x, y$ coordinate position based information, where $x_{p}$ and $y_{p}$ are predicted location of neighbor nodes at $(x, y)$ co-ordinate and $T_{c}$ is the time when the node decides to forward a packets to one of its neighboring node. The current location of a given neighbor nodes $\left(x_{p}, y_{p}\right)$ 


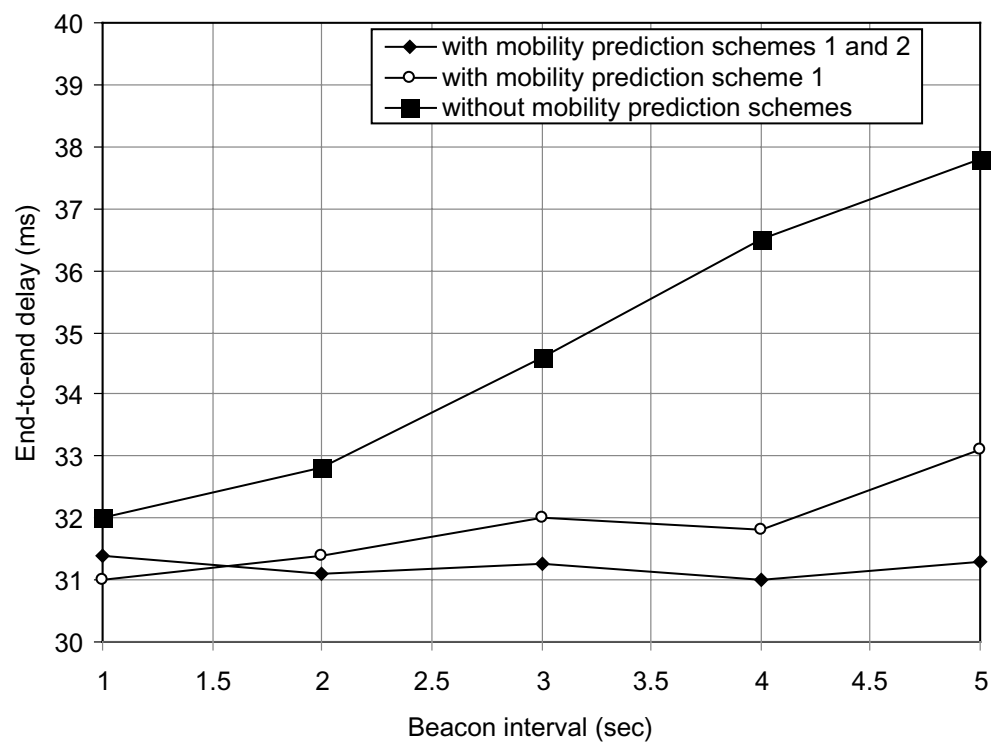

Fig. 13. End-to-end delay varying beacon interval.

is predicted whenever a node looks up a neighbors list for routing decision made by source node based on the calculated nodes location. The source node now can calculate its distance from the predicted neighbor nodes location using the Pythagorean Theorem,

$$
D_{p}^{i}=\sqrt{x_{p}^{i 2}+y_{p}^{i 2}}
$$

Where $D_{p}$ is the predicted neighbor nodes distance from source node.

By using the two prediction schemes, the source node has the ability to avoid forwarding the packet to a node that is located outside its transmission range even though it is still listed in its neighbors list as a neighbor node.

\subsection{Result and discussion}

Among the tested mobility models, we used BSA mobility model to implement our prediction schemes. BSA brought good results in less number of packets drop when the beacon interval and node speed increase as shown in Figs 5 and 9 respectively. In addition, it has already been criticized in $[2,8]$ that RWP and RD mobility models may cause unrealistic movement behavior with sudden speed and direction change since new choice for speed and direction values is not correlated to previous values. BSA includes both autocorrelation features. Speed is changed incrementally by the current acceleration of the $\mathrm{MN}$, and also the change in direction is smooth: Once a station is intended to turn, the direction is changed in several time steps until the new target direction is achieved.

Figures 13 and 14 show the end-to-end delay when we the beacon interval and node speed got varied with and without mobility prediction schemes. The end-to-end delay is less even with longer beacon interval and higher node speed. The actual reason behind that is explained by IEEE 802.11 standard [12]. According to the standard, a maximum number of retransmissions are allowed before the packets are dropped. As if the sender does not receive the ACK frame within time interval for the sent packet, it retransmits the packet until it receives ACK frame or throws it away after maximum 


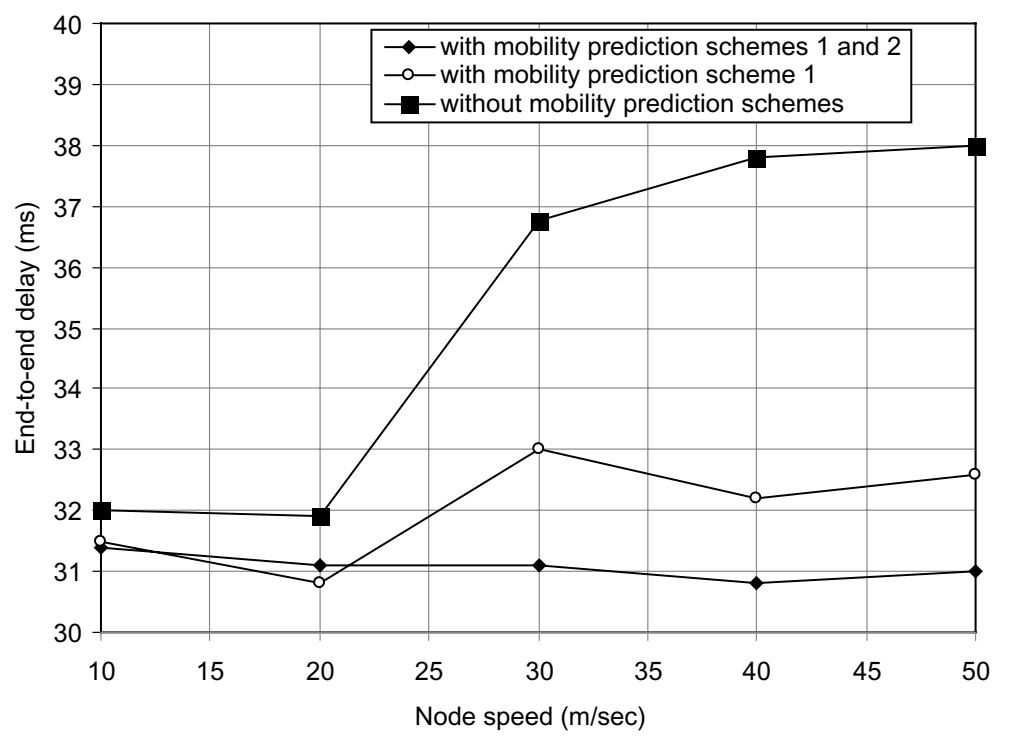

Fig. 14. End-to-end delay varying node speed.

number of retransmissions. The NBL problem causes the packet to be retransmitted several times to the neighbor that is listed in neighbors list but out of transmission range. That is, before the sender considers the neighbor could not be reached. The packet retransmission caused the packet to have additional propagation, transmission, and processing delay. However, by using mobility prediction schemes, the packet avoids these additional delays.

Another result shows in Figs 13 and 14 is that even when the mobility prediction scheme 1 is used; the end-to-end delay is considerably still increases. As it is explained in Fig. 11, this is due to the fact that when the beacon interval and node speed increase, the probability of node sending two beacon packets decreases. That is as the node may pass the boarder of other nodes transmission range sending just one beacon packet which will result in missing the use of the mobility prediction scheme 1 by sending node and it is overcome by using both mobility prediction schemes as it is shown in Figs 13, 14 also.

Figures 15 and 16 show the delivery ratio when the beacon interval and node speed got varied with and without mobility prediction schemes. The delivery ratio is up even with a longer beacon interval and a higher node speed. To identify the actual reason for this improvement, as it is discussed earlier, the mobility prediction schemes are used to reduce the number of NBL problems caused by inaccurate neighbor location information. Mobility prediction schemes are used to blacklist neighbor nodes that are estimated to be out of the communication range at the moment of packet forwarding. In this way, we avoid sending the packets to neighbors once they are out of the node's transmission range. That causes the delivery ratio to be higher.

\section{Conclusion and future work}

In this paper, we study the effect of inaccurate location information caused by mobility metrics; node speed and beacon interval on geographical GPSR mobile ad hoc network routing protocol. We identified the problem of NBL and discussed the mobility metrics that cause NBL problem. We also proposed prediction schemes to overcome NBL problem. By using prediction schemes, the source node has the 


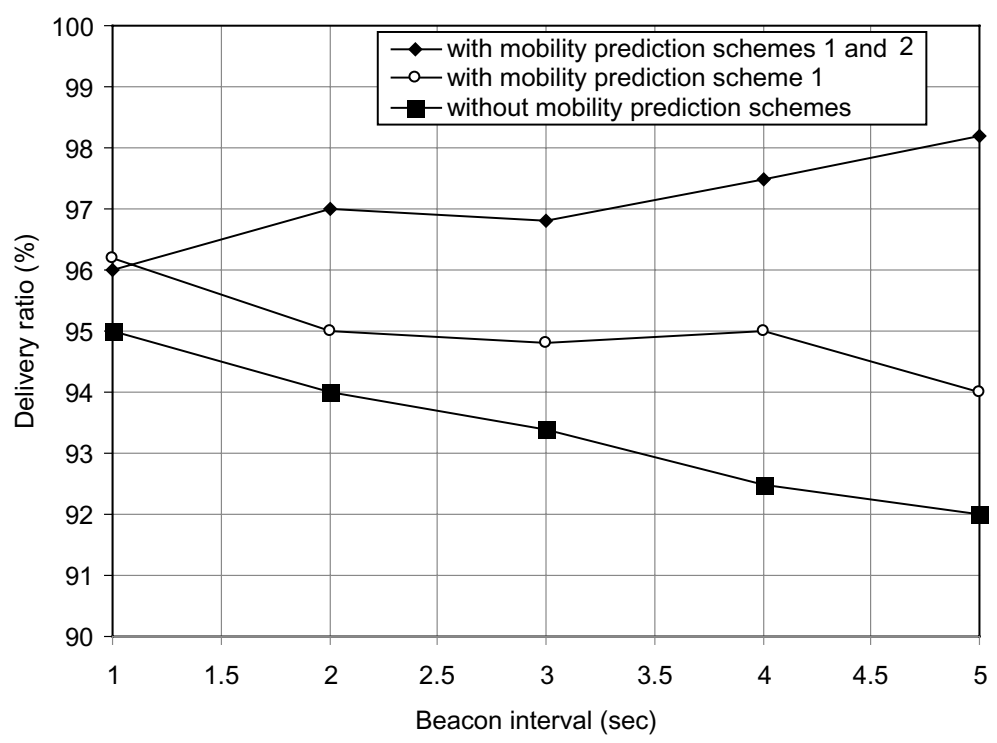

Fig. 15. Delivery ratio varying beacon interval.

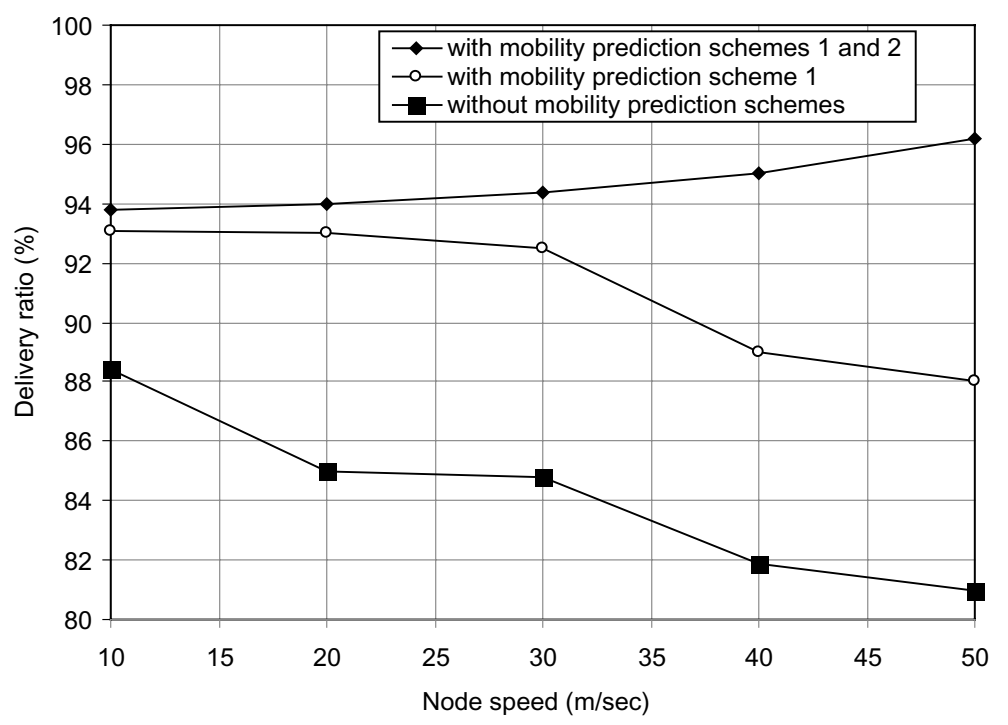

Fig. 16. Delivery ratio varying node speed.

ability to avoid forwarding the packet to a node that is located outside its transmission range even though it is still listed in its neighbors list as a neighbor node, which leads to improve the delivery ratio and the end-to-end delay.

In our future work, we aim to adapt the periodic beaconing strategy of the position-based routing protocols and improve the reliability of node's neighbors list by optimizing the time interval between the transmissions of beacon packets. The optimization will be based on the correlation between the node mobility and periodic beaconing of beacon packet transmission. The high change in topology which is caused by nodes movement should increase the number of beacon packets. That is to get an accurate 
node's neighbor list in order to assist the node to make suitable routing decision.

\section{References}

[1] B. Karp, Geographical Routing for Wireless Networks, PhD Dissertation, Harvard University, October 2000.

[2] C. Bettstetter, Mobility modeling in wireless networks: categorization, smooth movement, and border effects, $A C M$ SIGMOBILE Mobile Computing and Communications Review, 5(3) (2001), 55-66, ACM Press .

[3] C. Maihofer, A Survey of Geocasting Routing Protocols, The Electronic Magazine of Original Peer-Reviewed Survey Articles, IEEE Communications Surveys \& Tutorials 6(2), Second Quarter 2004.

[4] D. Turgut, S.K. Das and M. Chatterjee, Longevity of Routes in Mobile Ad Hoc Networks, (Vol. 4), 53rd IEEE Vehicular Technology Conference (VTS'01 Spring), 2001, 2833-2837.

[5] E. Royer and C.-K. Toh, A Review of Current Routing Protocols for Ad Hoc Wireless Networks, IEEE Pers Communication (Apr. 1999), 46-55.

[6] H. Larkin, Z. Wu and W. Toomey, Mapping Wireless Signal Strength for Mobile Adhoc Networks, Proceedings of the International Conference on Wireless Networks (ICWN'04), Las Vegas, USA. CSREA Press, 2004, 151-157.

[7] H. Larkin, Z. Wu and W. Toomey, Predicting Network Topology for Autonomous Wireless Nodes, in European Wireless, 2005, pp. 413-417.

[8] H.M. Gerla, G. Pei and C.-C. Chiang, A Group Mobility Model for Ad Hoc Wireless Networks, Proceeding of International Workshop on Modeling, Analysis, and Simulation of Wireless and Mobile Systems, Seattle, USA, 1999.

[9] H. Samarth, S. Nahrstedt and K, Nahrstedt, Predictive Location-Based QoS Routing in Mobile Ad Hoc Networks, (Vol. 2), IEEE International Conference on Communications, ICC 2002, 28 April-2 May 2002, 1022-1027.

[10] H. Takagi and L. Kleinrock, Optimal transmission ranges for randomly distributed packet radio terminals, IEEE Transactions on Communications 32(3) (1984), 246-257.

[11] I.D. Chakeres and M.B. Elizabeth, The Utility of Hello Messages for Determining Link Connectivity, Proceedings of the 5th International Symposium on Wireless Personal Multimedia Communications (WPMC) 2002, Honolulu, Hawaii, October 2002.

[12] IEEE Std. 802.11, Wireless LAN Media Access Control (MAC) and Physical Layer (PHY) Specification, 1999.

[13] J. Tang, G. Xue and W. Zhang, Reliable Ad Hoc Routing Based on Mobility Prediction, Journal of Combinatorial Optimization 11(1) (Feb 2006).

[14] J. Tsumochi, K. Masayama, H. Uehara and M. Yokoyama, Impact of Mobility Metric on Routing Protocols for Mobile Ad Hoc Networks, (Vol. 1), IEEE Pacific Rim Conference on Communication, Computers and Signal Processing, PACRIM 2003, 28-30 Aug 2003, 322-325.

[15] M. Heissenbüttel, T. Braun, M. Wälchli and T. Bernoulli, Evaluating the limitations of and alternatives in beaconing, Elsevier Science Publishers B. V. 5(5) (July 2007), 558-578.

[16] M. Mauve, J. Widmer and H. Hartenstein, A Survey on Position-Based Routing in Mobile Ad Hoc networks, IEEE Network 1(6) (Dec. 2001), 30-39.

[17] S.J. Philip, Scalable Location Management for Geographical Routing in Mobile Ad Hoc Networks, Ph.D thesis, The State University of New York, Buffalo, 2005.

[18] W. Su, Motion Prediction in Mobile/Wireless Networks, PhD Thesis. University of California, Los Angeles, USA, 2000.

[19] T. Camp, J. Boleng and V. Davies, A survey of mobility models for ad hoc network research, Wireless Communications $\&$ Mobile Computing (WCMC): Special issue on Mobile Ad Hoc Networking: Research, Trends and Applications 2(5) (2002), 483-502.

Raed Saqour received his B.Sc. degree in computer science from Mu'tah University, Jordan, in 1997. He obtained his M.Sc (Hons) degree in Distributed System from University Putra Malaysia, UPM, Malaysia, in 2003. He expected to receive his Ph.D. degree in Wireless Communication System in May 2008 from Universiti Kebangsaan Malaysia, UKM, Malaysia. He is a graduate student researcher. His research interests include wireless network, Ad hoc network, routing protocols, and network performance evaluation. E-mail:r_saqour2002@yahoo.com.

Mohamad Shanudin is an Associate Professor in the Faculty of Information Science and Technology and coordinator of postgraduate studies at Universiti Kebangsaan Malaysia (Malaysia). He received his B.Sc., M.Sc., in Computer Science from Northrop University, California, in 1984, and 1985, respectively. He obtained his Ph.D. from University of Reading, UK, in 1994, his area of research include Mobile Network (adhoc, IPv6, IPSec), knowledge Management, and Agent-based e-Commerce. E-mail: msz@ftsm.ukm.my.

Mahamod Ismail is currently an Associate Professor with the Department of Electrical, Electronics and System Engineering, Universiti Kebangsaan Malaysia. He received the B.Sc. degree in Electrical and Electronics from University of Strathclyde, 
U.K. in 1985, the M.Sc. degree in Communication Engineering and Digital Electronics from UMIST, Manchester U.K. in 1987, and the Ph.D. from University of Bradford, U.K. in 1996. His research interests include mobile communication and wireless networking particularly on the radio resource management. He is the past chapter chair of IEEE Communication Society and IEEE Malaysia Educational Activities. E-mail: mahamod@eng.ukm.my. 

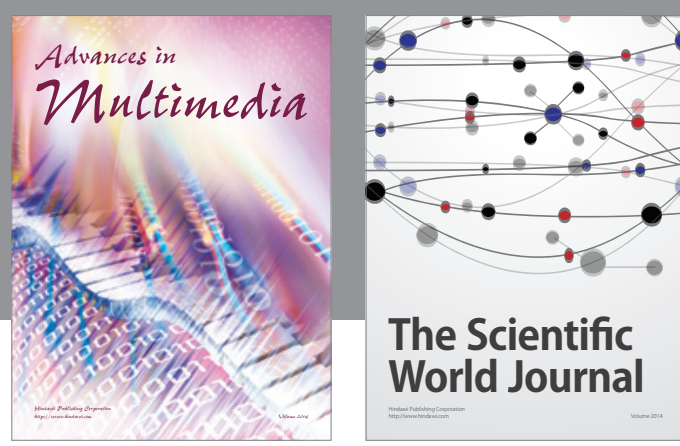

The Scientific World Journal
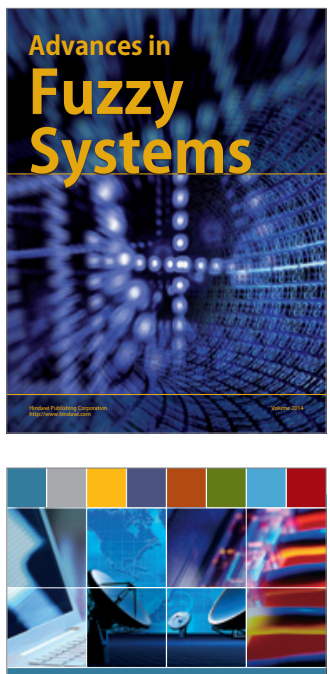

Computer Networks and Communications
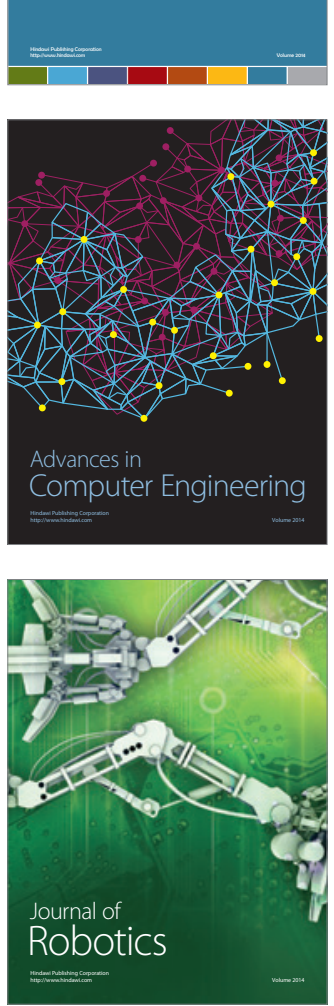
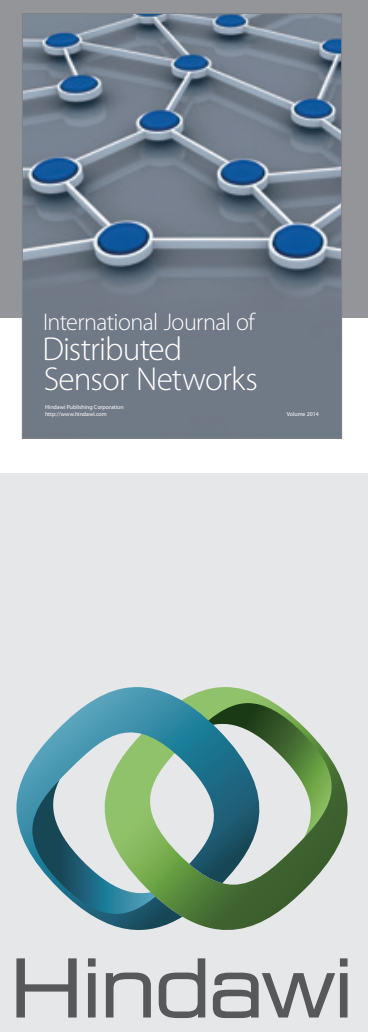

Submit your manuscripts at

http://www.hindawi.com
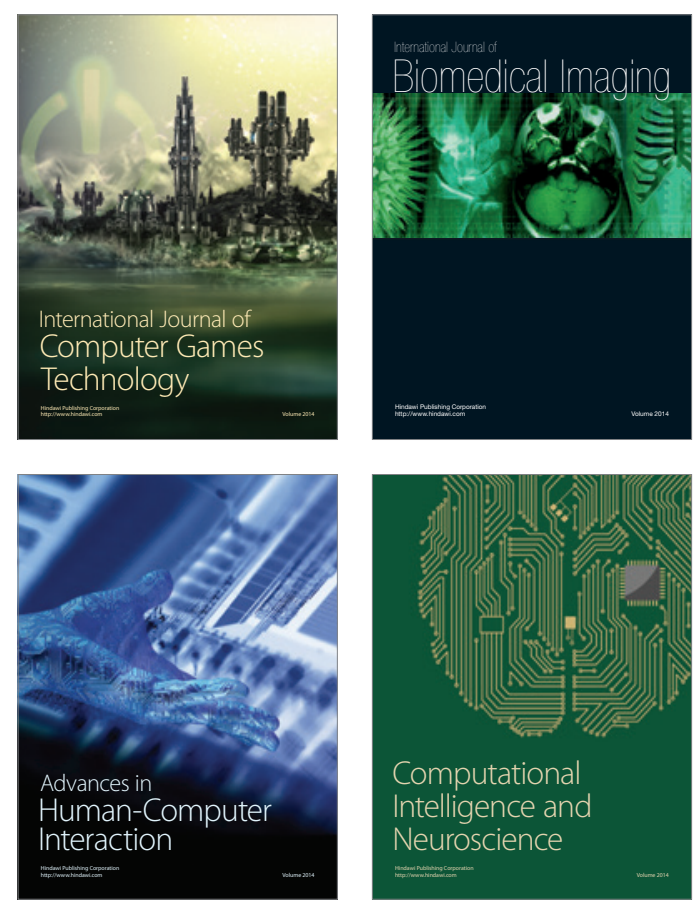
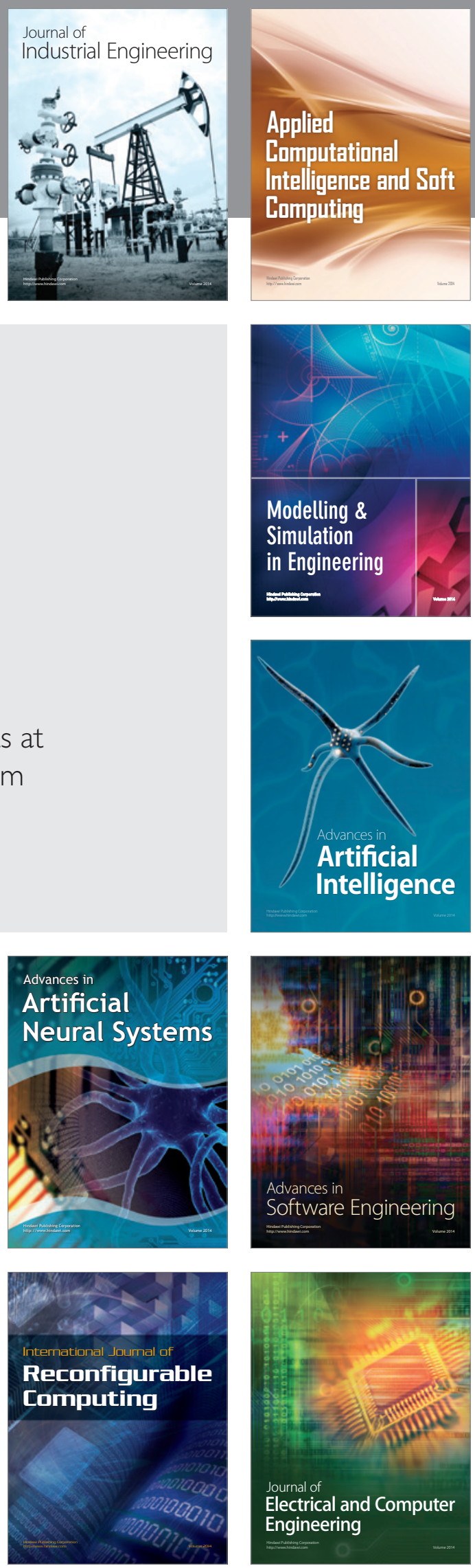